\title{
Complex convexity and vector-valued Littlewood-Paley inequalities
}

\author{
Oscar Blasco*, Miroslav Pavlović*
}

\begin{abstract}
Let $2 \leq p<\infty$ and let $X$ be a complex Banach space. It is shown that $X$ is $p$-uniformly $P L$-convex if and only if there exists $\lambda>0$ such that $\|f\|_{H^{p}(X)} \geq\left(\|f(0)\|^{p}+\lambda \int_{\mathbb{D}}\left(1-|z|^{2}\right)^{p-1}\left\|f^{\prime}(z)\right\|^{p} d A(z)\right)^{1 / p}$, for all $f \in H^{p}(X)$. Applications to embeddings between vector-valued $B M O A$ spaces defined via Poisson integral or Carleson measures are provided.
\end{abstract}

AMS Subject Class. 46B20,46L52

\section{Introduction}

Throughout the paper $X$ will always be a complex Banach space, $d A(z)=$ $\frac{1}{\pi} r d r d \theta$ stands for the normalized Lebesgue measure in the unit disc $\mathbb{D}$, we write $M_{p}(r, f)=\left(\frac{1}{2 \pi} \int_{0}^{2 \pi}\left\|f\left(r e^{i t}\right)\right\|^{p} d t\right)^{1 / p}$ for $1 \leq p<\infty$ and $H^{p}(X)$ stands for the space of analytic functions in $\mathbb{D}$ with values in $X$ such that $\sup _{0<r<1} M_{p}(r, f)<\infty$.

When extending the notions of modulus of convexity to complex Banach spaces, different complex convexities have been considered in the literature. Recall the following moduli: If $1 \leq p \leq \infty$

$$
H_{\infty}^{X}(\epsilon)=\inf \left\{\sup _{\theta}\left\|x+e^{i \theta} y\right\|-1:\|x\|=1,\|y\|=\epsilon\right\}
$$

\footnotetext{
*The first author has been partially supported by DGICYT under Proyecto PB98-0146
} 


$$
H_{p}^{X}(\epsilon)=\inf \left\{\left(\frac{1}{2 \pi} \int_{0}^{2 \pi}\left\|x+e^{i \theta} y\right\|^{p} d \theta\right)^{1 / p}-1:\|x\|=1,\|y\|=\epsilon\right\}
$$

A complex Banach space is called uniformly $\mathbb{C}$-convex (see [10]), also called uniformly $H^{\infty}$-convex (see [8]), if $H_{\infty}^{X}(\epsilon)>0$ for all $\epsilon>0$ and uniformly $P L$-convex (see [8]) if $H_{1}^{X}(\epsilon)>0$ for all $\epsilon>0$

It is known that $H_{1}^{X}(\epsilon)>0$ for all $\epsilon>0$ if and only if $H_{\infty}^{X}(\epsilon)>0$ for all $\epsilon>0$. Actually (see [9]) there exists $A>0$ such that

$$
A\left(H_{\infty}^{X}(\epsilon)\right)^{2} \leq H_{1}^{X}(\epsilon) \leq H_{\infty}^{X}(\epsilon) .
$$

When these moduli are of power type we have the following notions: A Banach space $X$ is said to be $p$-uniformly $\mathbb{C}$-convex $(p \geq 2)$ (see [10]) there is a positive constant $\lambda$ such that

$$
\max _{\theta}\left\|x+e^{i \theta} y\right\| \geq\left(\|x\|^{p}+\lambda\|y\|^{p}\right)^{1 / p}
$$

for all $x, y \in X$.

$X$ is called $p$-uniformly $P L$-convex (see [8]) if there is a positive constant $\lambda$ such that

$$
\frac{1}{2 \pi} \int_{0}^{2 \pi}\left\|x+e^{i \theta} y\right\|^{p} d \theta \geq\|x\|^{p}+\lambda\|y\|^{p}
$$

for all $x, y \in X$ for some constant $\lambda>0$. We write $I_{p}(X)$ for the largest possible value of $\lambda$.

It is known that, for each fixed $p$, these notions are equivalent (see [11]).

It is not difficult to see that $X$ is $p$-uniformly $P L$-convex if and only if $X$ has modulus of $P L$-convexity of power type $p$, i.e. there exists $\delta>0$ such that $H_{p}^{X}(\epsilon) \geq \delta \epsilon^{p}$ for $\epsilon>0$ (see the argument in Proposition 2.2 for a proof).

Let us also mention that if $X$ is $p$-uniformly $P L$-convex then also $L^{p}(\Omega, X)$ has the same property (see [8]).

Another complex convexity, introduced in [15], is the following: A Banach space $X$ is said to be uniformly $H_{p}$-convex if $h_{p}^{X}(\epsilon)>0$ for all $\epsilon>0$ where

$$
h_{p}^{X}(\epsilon)=\inf \left\{\|f\|_{H^{p}(X)}-1:\|f(0)\|=1,\|f-f(0)\|_{H^{p}(X)} \geq \epsilon\right\} .
$$

Since $H_{p}^{X}(\epsilon) \geq h_{p}^{X}(\epsilon)$, any uniformly $H_{p}$-convex space is also uniformly $P L$-convex. 
A Banach space $X$ is called $p$-uniformly $H_{p}$-convex (see $\left.[14,12,16,17]\right)$ if there is a positive constant $\lambda$ such that

$$
\|f\|_{H^{p}(X)} \geq\left(\|f(0)\|^{p}+\lambda\|f-f(0)\|_{H^{p}(X)}\right)^{1 / p},
$$

for all $f \in H^{p}(X)$. We denote by $J_{p}(X)$ the largest possible value of $\lambda$.

Of course, if $X$ is $p$-uniformly $H_{p}$-convex then $X$ is $p$-uniformly $P L$-convex and $J_{p}(X) \leq I_{p}(X)$.

Vector-valued versions of Littlewood-Paley theory have been considered by several authors and several reasons. Let us mention the notion we shall be using in this paper.

Let $2 \leq p<\infty$. A complex Banach space is said to have analytic Lusin cotype $p$ (see [17]) (called also property $(H)_{p}$ in [1]) if there exist $C>0$ such that

$$
\left(\int_{\mathbb{D}}\left(1-|z|^{2}\right)^{p-1}\left\|f^{\prime}(z)\right\|^{p} d A(z)\right)^{1 / p} \leq C\|f\|_{H^{p}(X)}
$$

for all $X$-valued polynomials $f$. For the use of this property and related ones we refer the reader to $[1,2,3,4,5,6,17]$.

It is known that analytic Lusin cotype $p$ implies cotype $p$ (see [1]). A remarkable fact, proved by $\mathrm{Q}$. $\mathrm{Xu}$, is its connection with complex convexity.

Theorem 1.1 (see [17], Theorem 5.1)

(i) If $X$ is p-uniformly $H^{p}$-convex then $X$ has analytic Lusin cotype $p$.

(ii) If $X$ has analytic Lusin cotype $p$ then $X$ can be renormed to have an equivalent p-uniformly PL-convex norm.

It was conjectured there that actually analytic Lusin cotype $p$ should coincide with $p$-uniformly $H^{p}$-convexity.

Our Corollary 2.4 establishes that this is not the case, actually we show that if $X$ has a $p$-uniformly $P L$-convex norm then $X$ has analytic Lusin cotype .

It should be pointed out that while Xu's arguments are based upon some martingale estimates, our proof of the converse of (ii) is mainly based upon subharmonic functions. In our arguments Riesz measures of the subharmonic functions $\|f\|^{p}$ where $f: \mathbb{D} \rightarrow X$ are analytic play an important role. The reader is referred to the recent paper by G. Blower and T. Ransford (see [7]) 
for the use of Riesz measures to determine the q-uniform PL-convexity of the spaces.

Let us now introduce a new modulus of complex convexity that we need for our proof.

Definition 1.2 Let $X$ be a complex Banach space and let $2 \leq p<\infty$. We define

$A_{p}^{X}(\epsilon)=\inf \left\{\|f\|_{H^{p}(X)}-1:\|f(0)\|=1,\left(\int_{\mathbb{D}}\left(1-|z|^{2}\right)^{p-1}\left\|f^{\prime}(z)\right\|^{p} d A(z)\right)^{1 / p} \geq \epsilon\right\}$,

Of course there exists $C>0$ such that $H_{p}^{X}(\epsilon) \geq A_{p}^{X}(C \epsilon)$ for all $\epsilon>0$.

Our main theorem establishes that $A_{p}^{X}$ is of power type $p$ if and only if the same happens to $H_{p}^{X}$. As a simple consequence we get that $p$-uniformly $P L$-convex spaces do have analytic Lusin cotype $p$.

The paper is divided into two sections. The main theorem and its proof is done in section 2 while the last section is devoted to get some applications. It is shown that if $X$ is a $p$-uniformly $P L$-convex space then $H^{p}(X)$ satisfies certain lacunary $p$-lower estimates (in the sense introduced by G. Pisier in [12]) and also that for 2-uniformly $P L$-convex spaces we have the embedding between vector-valued BMOA spaces defined by Carleson measures and the one defined by the condition $\sup _{z \in \mathbb{D}} P\left(\|f\|^{2}\right)(z)-\|f(z)\|^{2}<\infty$.

\section{The main theorem}

Let us start by characterizing $p$-uniformly $P L$-convex spaces in terms of vector valued Hardy spaces.

Proposition 2.1 Let $X$ be a complex Banach space and $2 \leq p<\infty$. The following are equivalent.

(i) $X$ is p-uniformly $P L$-convex.

(ii) There exists a constant $c>0$ such that

$$
\|f\|_{H^{\infty}(X)} \geq\left(\|f(0)\|^{p}+c\left\|f^{\prime}(0)\right\|^{p}\right)^{1 / p},
$$

for all $f \in H^{\infty}(X)$. 
(iii) There exists a constant $c>0$ such that

$$
\|f\|_{H^{p}(X)} \geq\left(\|f(0)\|^{p}+c\left\|f^{\prime}(0)\right\|^{p}\right)^{1 / p}
$$

for all $f \in H^{p}(X)$.

Proof. (i) $\Rightarrow$ (ii). Let $\|f\|_{H^{\infty}(X)}=1$ and $\phi \in X^{*},\|\phi\|=1$. By Schwarz' lemma,

$$
\left|\phi\left(f^{\prime}(0)\right)\right| \leq 1-|\phi(f(0))|^{2} \leq 2(1-|\phi(f(0))|) .
$$

Hence for all $\theta$

$$
\left|\phi\left(f(0)+e^{i \theta} f^{\prime}(0) / 2\right)\right| \leq|\phi(f(0))|+(1 / 2)\left|\phi\left(f^{\prime}(0)\right)\right| \leq 1,
$$

and, therefore $\left\|f(0)+e^{i \theta} f^{\prime}(0) / 2\right\| \leq 1$.

Now since $X$ is $p$-uniformly $P L$-convex we get $(i i)$ with $c=I_{p}(X) / 2^{p}$.

(ii) $\Rightarrow$ (i). It is obvious that (ii) implies that $X$ is $p$-uniformly $\mathbb{C}$-convex. Hence also $p$-uniformly $P L$-convex using [11].

(i) $\Rightarrow$ (iii) Assume that $f \in H^{p}(X)$ and define the analytic function $g$ : $\mathbb{D} \mapsto L^{p}(\partial \mathbb{D}, X)=: Y$ by $g(z)\left(e^{i \theta}\right)=f\left(z e^{i \theta}\right) \quad(z \in \mathbb{D})$. Then $g \in H^{\infty}\left(L^{p}(X)\right)$ and $\|g\|_{H^{\infty}\left(L^{p}(X)\right)}=\|f\|_{H^{p}(X)}$. Hence we get (iii) with $c=I_{p}\left(L^{p}(X)\right) / 2^{p}$.

(iii) $\Rightarrow(\mathrm{i})$. This is obvious.

Let us now present an equivalent formulation for the modulus $A_{p}^{X}$ to be of power type $p$.

Proposition 2.2 Let $X$ be complex Banach space and $2 \leq p<\infty$. The following are equivalent.

(i) There exists $\lambda>0$ such that

$$
\|f\|_{H^{p}(X)} \geq\left(\|f(0)\|^{p}+\lambda \int_{\mathbb{D}}\left(1-\|z\|^{2}\right)^{p-1}\left\|f^{\prime}(z)\right\|^{p} d A(z)\right)^{1 / p}
$$

for all $f \in H^{p}(X)$.

(ii) There exists $\delta>0$ such that $A_{p}^{X}(\epsilon) \geq \delta \epsilon^{p}$ for all $\epsilon>0$.

Proof. Assume (i). For each function $f \in H^{p}(X)$ such that $\|f(0)\|=1$ and $\int_{\mathbb{D}}\left(1-|z|^{2}\right)^{p-1}\left\|f^{\prime}(z)\right\|^{p} d A(z) \geq \epsilon^{p}$ we have

$$
\|f\|_{H^{p}(X)}-1 \geq\left(1+\lambda \epsilon^{p}\right)^{1 / p}-1 \geq(\lambda / p) \epsilon^{p} .
$$


Hence $A_{p}^{X}(\epsilon) \geq(\lambda / p) \epsilon^{p}$.

On the other hand if $A_{p}^{X}(\epsilon) \geq \delta \epsilon^{p}$ for $\epsilon>0$ then for any $x \in X \backslash\{0\}$ one has

$$
\begin{aligned}
\|f\|_{H^{p}(X)}-\|f(0)\| & =\|f(0)\|\left(\left\|\frac{f}{\|f(0)\|}\right\|_{H^{p}(X)}-1\right) \\
& \geq \delta\|f(0)\|^{1-p}\left(\int_{\mathbb{D}}\left(1-|z|^{2}\right)^{p-1}\left\|f^{\prime}(z)\right\|^{p} d A(z)\right) .
\end{aligned}
$$

This shows that

$$
\begin{aligned}
\|f\|_{H^{p}(X)} & \geq\|f(0)\|\left(1+\delta \int_{\mathbb{D}}\left(1-|z|^{2}\right)^{p-1} \frac{\left\|f^{\prime}(z)\right\|^{p}}{\|f(0)\|^{p}} d A(z)\right) \\
& \geq\left(\|f(0)\|^{p}+\delta \int_{\mathbb{D}}\left(1-|z|^{2}\right)^{p-1}\left\|f^{\prime}(z)\right\|^{p} d A(z)\right)^{1 / p} .
\end{aligned}
$$

This shows that if $A_{p}^{X}(\epsilon) \geq \delta \epsilon^{p}$ for some $\delta>0$ then $X$ is $p$-uniformly $P L$-convex. Our main result establishes that the converse is also true.

Theorem 2.3 Let $2 \leq p<\infty$. The following are equivalent.

(i) $X$ is p-uniformly $P L$-convex.

(ii) There exists a constant $C>0$ such that for each $r<\frac{1}{2}$

$$
\left\|f^{\prime}(z)\right\|^{p}\left(1-|z|^{2}\right)^{p} \leq \frac{C}{r^{p}\left(1-r^{2}\right)^{p}} \int_{D_{2 r}(z)} d \mu(w)
$$

where $d \mu$ is the Riesz measure of the subharmonic function $\|f\|^{p}$ (see [13]) and $D_{r}(z)$ denotes the ball centred at $z$ and radius $r$ for the pseudo-hyperbolic distance $d(w, z)=\left|\phi_{z}(w)\right|=\left|\frac{z-w}{1-\bar{z} w}\right|$.

(iii) There exists $\lambda>0$ such that

$$
\|f\|_{H^{p}(X)} \geq\left(\|f(0)\|^{p}+\lambda \int_{\mathbb{D}}\left(1-|z|^{2}\right)^{p-1}\left\|f^{\prime}(z)\right\|^{p} d A(z)\right)^{1 / p}
$$

for all $f \in H^{p}(X)$.

Proof. (i) $\Rightarrow$ (ii) Let us fix $0<r<1$. If $u$ is an arbitrary subharmonic function on $\mathbb{D}$ then (Poisson-Jensen) formula states that

$$
\frac{1}{2 \pi} \int_{0}^{2 \pi} u\left(r e^{i \theta}\right) d \theta-u(0)=\frac{1}{2 \pi} \int_{|w|<r} \log \frac{r}{|w|} d \mu(w) .
$$


where $\mu$ stands for the Riesz measure associated to the subharmonic function $u$ (see [13]).

Applying this to $u(z)=\|f(z)\|^{p}$ and using (2.2) in Proposition 2.1 for the function $f_{r}(z)=f(r z)$ we get

$$
r^{p}\left\|f^{\prime}(0)\right\|^{p} \leq \frac{1}{\lambda} \int_{|w|<r} \log \frac{r}{|w|} d \mu(w) .
$$

Applying (2.4) to $f\left(\phi_{\xi}(w)\right)$ for $\phi_{\xi}(w)=\frac{\xi-w}{1-\bar{\xi} w}$ we obtain

$$
r^{p}\left(1-|\xi|^{2}\right)^{p}\left\|f^{\prime}(\xi)\right\|^{p} \leq \frac{1}{\lambda} \int_{D_{r}(\xi)} \log \frac{r}{\left|\phi_{\xi}(w)\right|} d \mu(w)
$$

On the other hand $\left\|f^{\prime}(0)\right\|^{p} \leq \frac{1}{r^{2}} \int_{|w|<r}\left\|f^{\prime}(w)\right\|^{p} d A(w)$, which implies

$$
\begin{aligned}
\left(1-|z|^{2}\right)^{p}\left\|f^{\prime}(z)\right\|^{p} & \leq \frac{1}{r^{2}} \int_{|w|<r}\left\|f^{\prime}\left(\phi_{z}(w)\right)\right\|^{p} \frac{\left(1-\left|\phi_{z}(w)\right|^{2}\right)^{p-2}}{\left(1-|w|^{2}\right)^{p-2}}\left|\phi_{z}^{\prime}(w)\right|^{2} d A(w) \\
& \leq \frac{1}{r^{2+p}\left(1-r^{2}\right)^{p-2}} \int_{D_{r}(z)} r^{p}\left(1-|\xi|^{2}\right)^{p-2}\left\|f^{\prime}(\xi)\right\|^{p} d A(\xi) .
\end{aligned}
$$

Now using (2.5) together with the previous estimate we obtain that again

$$
\begin{aligned}
& \left(1-|z|^{2}\right)^{p}\left\|f^{\prime}(z)\right\|^{p} \leq \frac{1}{\lambda} \frac{1}{r^{2+p}\left(1-r^{2}\right)^{p-2}} \int_{D_{r}(z)}\left(\int_{D_{r}(\xi)} \log \frac{r}{\left|\phi_{\xi}(w)\right|} d \mu(w)\right) \frac{d A(\xi)}{\left(1-|\xi|^{2}\right)^{2}} \\
& \leq \frac{1}{\lambda} \frac{1}{r^{2+p}\left(1-r^{2}\right)^{p-2}} \int_{D_{2 r}(z)}\left(\int_{D_{r}(w)} \log \frac{r}{\left|\phi_{w}(\xi)\right|} \frac{d A(\xi)}{\left(1-|\xi|^{2}\right)^{2}}\right) d \mu(w) \\
& \leq \frac{1}{\lambda} \frac{1}{r^{2+p}\left(1-r^{2}\right)^{p-2}} \int_{D_{2 r}(z)}\left(\int_{|\xi|<r} \log \frac{r}{|\xi|} \frac{d A(\xi)}{\left(1-|\xi|^{2}\right)^{2}}\right) d \mu(w)
\end{aligned}
$$

Using now that $\int_{|\xi|<r} \log \frac{r}{|\xi|} \frac{d A(\xi)}{\left(1-|\xi|^{2}\right)^{2}} \leq \frac{r^{2}}{\left(1-r^{2}\right)^{2}} \int_{|\xi|<1} \log \frac{1}{|\xi|} d A(\xi)=\frac{r^{2}}{2\left(1-r^{2}\right)^{2}}$ we get (ii) with $C=\frac{1}{2 \lambda}$.

(ii) $\Rightarrow$ (iii) Fix $r=\frac{1}{4}$ and let $f \in H^{p}(X)$. Integrating and applying Fubini we get

$$
\int_{\mathbb{D}}\left\|f^{\prime}(z)\right\|^{p}\left(1-|z|^{2}\right)^{p-1} d A(z) \leq C \int_{\mathbb{D}}\left(\int_{D_{1 / 2}(w)}\left(1-|z|^{2}\right)^{-1} d A(z)\right) d \mu(w) .
$$


Since $\int_{D_{1 / 2}(w)}\left(1-|z|^{2}\right)^{-1} d A(z) \leq C(1-|w|)$, we get

$$
\begin{aligned}
\int_{\mathbb{D}}\left\|f^{\prime}(z)\right\|^{p}\left(1-|z|^{2}\right)^{p-1} d A(z) & \leq C \int_{\mathbb{D}}(1-|w|) d \mu(w) \\
& \leq C \int_{\mathbb{D}} \log \frac{1}{|w|} d \mu(w) \\
& =C \lim _{r \rightarrow 1} \int_{|w|<r} \log \frac{r}{|w|} d \mu(w) \\
& =C \lim _{r \rightarrow 1}\left(\frac{1}{2 \pi} \int_{0}^{2 \pi}\left\|f\left(r e^{i \theta}\right)\right\|^{p} d \theta-\|f(0)\|^{p}\right) .
\end{aligned}
$$

(iii) $\Rightarrow$ (i) This is obvious.

Corollary 2.4 Let $2 \leq p<\infty$. Then $X$ has analytic Lusin cotype $p$ if and only if $X$ has an equivalent norm with respect to which it is p-uniformly $P L$-convex.

Proof. Theorem 2.3 gives one implication and the other corresponds to Theorem 5.1(ii) in [17] .

Corollary 2.5 Let $p \geq 2,1 \leq q \leq p$ and let $X$ be a $p$-uniformly $P L$-convex space. If $f \in H^{q}(X)$, then

$$
\int_{0}^{1}\left\{M_{q, X}\left(r, f^{\prime}\right)\right\}^{p}(1-r)^{p-1} d r \leq C\left(\|f\|_{H^{q}(X)}^{p}-\|f(0)\|^{p}\right) .
$$

Proof. The space $Y=L^{q}(\partial \mathbb{D}, X)$ is $p$-uniformly $P L$-convex. Applying Theorem 2.3 to the function $g(z)\left(e^{i \theta}\right)=f\left(e^{i \theta} z\right)$ we get the result.

It should be noted that inequality (2.6) contains, besides the LittlewoodPaley inequality, the inequality of Hardy and Littlewood,

$$
\int_{0}^{1}\left\{M_{q}\left(r, f^{\prime}\right)\right\}^{2}(1-r) d r \leq C\left(\|f\|_{q}^{p}-|f(0)|^{p}\right) \quad(1 \leq q \leq 2),
$$

which holds for scalar-valued $H^{q}$-functions.

Theorem 2.3 can be generalized, with the same proof, to obtain the following.

Theorem 2.6 Suppose that $X$ is p-uniformly $P L$-convex and that $p \leq q<$ $\infty$. If $f \in H^{q}(X)$ then

$$
\left.\int_{\mathbb{D}}\left\|f^{\prime}(z)\right\|^{p}\|f(z)\|^{q-p}(1-|z|)^{p-1} d A(z) \leq C\left(\|f\|_{q, X}^{q}-\|f(0)\|^{q}\right)\right) .
$$




\section{Applications}

Let us recall the following notion introduced by Pisier in [12] and closely related to $p$-uniform convexity.

Let $p \geq 2$ and $X$ be a complex Banach space. We say that $H^{p}(X)$ satisfies a radial lower $p$-estimate if there exist $\delta>0$ and $C \geq 1$ such that for all increasing sequences $0<r_{0}<r_{1}<\ldots<r_{n} \ldots<1$ and for all $f$ in $H^{p}(X)$ we have

$$
\left(\left\|f_{r_{0}}\right\|_{H^{p}(X)}^{p}+\delta \sum_{n=1}^{\infty}\left\|f_{r_{n}}-f_{r_{n-1}}\right\|_{H^{p}(X)}^{p}\right)^{1 / p} \leq C\|f\|_{H^{p}(X)}^{p}
$$

It was shown in [12] that if $X$ is $p$-uniformly convex then $H^{p}(X)$ satisfies a radial lower $p$-estimate with constant 1 .

We say that $H^{p}(X)$ satisfies a lacunary lower $p$-estimate (see [4]) if the previous estimate holds only for $r_{n}=1-2^{-n}$.

Now we can prove the following counterpart for $p$-uniformly $P L$-spaces.

Theorem 3.1 If $X$ is p-uniformly PL-convex then $H^{p}(X)$ satisfies a lacunary lower p-estimate with constant 1.

Proof. Let $r_{n}=1-2^{-n}$ and let $f$ be a function in $H^{p}(X)$. Since $f_{r_{n}}\left(e^{i t}\right)-$ $f_{r_{n}}\left(e^{i t}\right)=\int_{r_{n-1}}^{r_{n}} f^{\prime}\left(s e^{i t}\right) d s$ we have

$\left\|f_{r_{n}}-f_{r_{n-1}}\right\|_{H^{p}(X)} \leq \int_{r_{n-1}}^{r_{n}} M_{p}\left(f^{\prime}, s\right) d s \leq\left(r_{n}-r_{n-1}\right)^{1 / p^{\prime}}\left(\int_{r_{n-1}}^{r_{n}} M_{p}^{p}\left(f^{\prime}, s\right) d s\right)^{1 / p}$.

Hence, due to the equality $r_{n}-r_{n-1}=1-r_{n}$, we obtain

$$
\sum_{n=0}^{\infty}\left\|f_{r_{n}}-f_{r_{n-1}}\right\|_{H^{p}(X)}^{p} \leq \sum_{n=0}^{\infty} \int_{r_{n-1}}^{r_{n}}(1-s)^{p-1} M_{p}^{p}\left(f^{\prime}, s\right) d s .
$$

Therefore

$$
\sum_{n=0}^{\infty}\left\|f_{r_{n}}-f_{r_{n-1}}\right\|_{H^{p}(X)}^{p} \leq \int_{|w|>r_{0}}(1-|w|)^{p-1}\left|f^{\prime}(w)\right|^{p} \frac{d A(w)}{|w|} .
$$

Now Theorem 2.3 leads to

$$
\left(\|f(0)\|^{p}+\lambda \int_{\mathbb{D}}\left(1-|w|^{p-1}\right)\left\|f^{\prime}(w)\right\|^{p} d A(w)\right)^{1 / p} \leq\|f\|_{H^{p}(X)}
$$


for some $\lambda>0$.

Composing with the Möbious transform $\phi_{z}(w)=\frac{z-w}{1-\bar{z} w}$ we get

$$
\begin{gathered}
\left(\|f(z)\|^{p}+\lambda \int_{\mathbb{D}}\left(1-|w|^{2}\right)^{p-1}\left\|f^{\prime}\left(\phi_{z}(w)\right)\right\|^{p}\left|\phi_{z}^{\prime}(w)\right|^{p} d A(w)\right)^{1 / p} \leq \\
\leq\left(\frac{1}{2 \pi} \int_{0}^{2 \pi} \| f\left(\phi_{z}\left(e^{i t}\right) \|^{p} d t\right)^{1 / p} .\right.
\end{gathered}
$$

The change of variable $e^{i \theta}=\phi_{z}\left(e^{i t}\right)$ (hence $d t=P_{z}\left(e^{i \theta}\right) d \theta$ ) and the fact $\left|\phi_{z}^{\prime}(w)\right|=\frac{1-\left|\phi_{z}(w)\right|^{2}}{1-|w|^{2}}$ imply

$$
\begin{aligned}
\left\|f\left(r e^{i u}\right)\right\|^{p} & +\lambda \int_{\mathbb{D}}\left(1-|w|^{2}\right)^{p-1}\left\|f^{\prime}(w)\right\|^{p} P_{r e^{i u}}(w) d A(w) \leq \\
& \leq\left(\frac{1}{2 \pi} \int_{0}^{2 \pi}\left\|f\left(e^{i \theta}\right)\right\|^{p} P_{r e^{i u}}\left(e^{i \theta}\right) d \theta .\right.
\end{aligned}
$$

Hence

$$
\left\|f_{r}\right\|_{H^{p}(X)}^{p}+\lambda \int_{\mathbb{D}}\left(1-|w|^{2}\right)^{p-1}\left\|f^{\prime}(w)\right\|^{p} \frac{1-r^{2}}{1-r^{2}|w|^{2}} d A(w) \leq\|f\|_{H^{p}(X)}^{p} .
$$

Combining (3.2) and (3.3) and noticing that $\frac{1-r_{0}^{2}}{1-r_{0}^{2}|w|^{2}}>\frac{1}{1+r_{0}^{2}}$ for $|w|>r_{0}$ we obtain

$$
\left\|f_{r_{0}}\right\|_{H^{p}(X)}^{p}+\lambda \frac{1+r_{0}^{2}}{r_{0}} \sum_{n=0}^{\infty}\left\|f_{r_{n}}-f_{r_{n-1}}\right\|_{H^{p}(X)}^{p} \leq\|f\|_{H^{p}(X)}^{p} .
$$

Now the fact that $r_{0}+\frac{1}{r_{0}} \geq 2$ gives the result with $\delta=2 \lambda$.

Let us now apply the result to some get some embeddings between different vector valued BMOA spaces. if

A $X$-valued analytic function $f \in H^{1}(\mathbb{T}, X)$ is said to belong to $B M O A(X)$

$$
\sup _{|z|<1} \frac{1}{2 \pi} \int_{0}^{2 \pi}\left\|f\left(e^{i t}\right)-f(z)\right\|^{2} P_{z}\left(e^{i t}\right) d t<\infty
$$

where $P_{z}(w)=\frac{1-|z|^{2}}{|1-\bar{z} w|}$. 
The norm is given by $\|f\|_{H^{1}(X)}+\sup _{|z|<1}\left(\frac{1}{2 \pi} \int_{0}^{2 \pi}\left\|f\left(e^{i t}\right)-f(z)\right\|^{2} P_{z}\left(e^{i t}\right) d t\right)^{1 / 2}$. )if

A $X$-valued analytic function $f$ is said to belong to $B M O A_{\mathcal{C}}(X)$ (see [3]

$$
\sup _{|z|<1} \int_{\mathbb{D}}\left(1-|w|^{2}\right)\left\|f^{\prime}(w)\right\|^{2} P_{z}(w) d A(w)<\infty
$$

The norm is given by $\|f(0)\|+\sup _{|z|<1}\left(\int_{\mathbb{D}}\left(1-|w|^{2}\right)\left\|f^{\prime}(w)\right\|^{2} P_{z}(w) d A(w)\right)^{1 / 2}$.

It is known that if $B M O A(X) \subset B M O A_{\mathcal{C}}(X)$ then $X$ has cotype 2 (see [3])

Recall that in the scalar case we have

$$
\frac{1}{2 \pi} \int_{0}^{2 \pi}\left|f\left(e^{i t}\right)-f(z)\right|^{2} P_{z}\left(e^{i t}\right) d t=P\left(|f|^{2}\right)(z)-|f(z)|^{2}
$$

Using this, we still can define another $B M O A$ space.

A $X$-valued analytic function $f$ is said to belong to $B M O A_{\mathcal{P}}(X)$ if

$$
\sup _{|z|<1} P\left(\|f\|^{2}\right)(z)-\|f(z)\|^{2}<\infty
$$

The norm is given by $\|f(0)\|+\sup _{|z|<1}\left(P\left(\|f\|^{2}\right)(z)-\|f(z)\|^{2}\right)^{1 / 2}$.

Theorem 3.2 If $X$ is 2-uniformly $P L$-convex space then the natural inclusion map from $B M O A_{\mathcal{P}}(X)$ into $B M O A_{\mathcal{C}}(X)$ is bounded.

Proof. Using Theorem 2.3 we have that

$$
\left(\|f(0)\|^{2}+\lambda \int_{\mathbb{D}}\left(1-|w|^{2}\right)\left\|f^{\prime}(w)\right\|^{2} d A(w)\right)^{1 / 2} \leq\|f\|_{H^{2}(X)}
$$

for all function $f \in H^{2}(X)$.

Composing with the Moebius transform $\phi_{z}(w)=\frac{z-w}{1-\bar{z} w}$ we get

$$
\left(\|f(z)\|^{2}+\lambda \int_{\mathbb{D}}\left(1-|w|^{2}\right)\left\|f^{\prime}\left(\phi_{z}(w)\right)\right\|^{2}\left|\phi_{z}^{\prime}(w)\right|^{2} d A(w)\right)^{1 / 2} \leq\left(\frac{1}{2 \pi} \int_{0}^{2 \pi} \| f\left(\phi_{z}\left(e^{i t}\right) \|^{2} d t\right)^{1 / 2}\right.
$$

The change of variable $e^{i \theta}=\phi_{z}\left(e^{i t}\right)$ gives $d t=P_{z}\left(e^{i \theta}\right) d \theta$ and also $\left|\phi_{z}^{\prime}(w)\right|=$ $\frac{1-\left|\phi_{z}(w)\right|^{2}}{1-|w|^{2}}$ we obtain 


$$
\left(\|f(z)\|^{2}+\lambda \int_{\mathbb{D}}\left(1-|w|^{2}\right)\left\|f^{\prime}(w)\right\|^{2} P_{z}(w) d A(w)\right)^{1 / 2} \leq\left(\frac{1}{2 \pi} \int_{0}^{2 \pi}\left\|f\left(e^{i \theta}\right)\right\|^{2} P_{z}\left(e^{i \theta}\right) d \theta\right)^{1 / 2}
$$

This gives the desired estimate of norms $\|f\|_{B M O A_{c}} \leq \frac{1}{\sqrt{\lambda}}\|f\|_{B M O A_{\mathcal{P}}}$.

\section{References}

[1] J.L. Arregui, O. Blasco, Convolution of three functions by means of bilinear maps and applications, Ill. J. Math. 43(1999), 264-280.

[2] O. Blasco, Convolution by means of bilinear maps and applications, Contemporary Math. 232 (1998), 85-103.

[3] O. Blasco, Remarks on vector-valued BMOA and vector-valued multipliers, Positivity 4(2000), 339-356.

[4] O. Blasco, On the area function for $H\left(\sigma_{p}\right)$, Bull. Polish Acad. Sci. 44 (1996), 285-292.

[5] G. Blower, Quadratic integrals and factorization of linear operators II, J. London Math. Soc. 56 (1997), 333-346 .

[6] G. Blower, Multipliers of Hardy spaces, quadratic integrals and FoiasWilliams-Peller operators, Studia Math. 131 (1998), 179-188 .

[7] G. Blower, T. Ransford Complex uniform convexity and Riesz measures, To appear.

[8] W.J. Davis, D.J.H. Garling and N. Tomczak-Jaegermann, The complex convexity of quasi-normed spaces, J. Funct. Anal. 55(1984), 110-150.

[9] S.J. Dilworth, Complex convexity and the geometry of Banach spaces, Math. Proc. Camb. Phil. Soc 99 (1986), 495-506.

[10] J. Globevnik, On complex strict and uniform convexity, Proc. Amer. Math. Soc. 47(1975), 175-178.

[11] M. Pavlović, On the complex uniform convexity of quasi-normed spaces, Math. Balkanica (N.S.) 5(1991), 92-98. 
[12] G. Pisier, Factorization of operator valued analytic functions, Adv. Math. 93 (1992), 61-125.

[13] W.K. Hayman, P.B. Kennedy, Subharmonic functions, Academic Press, London (1976).

[14] U. Haagerup, G. Pisier, Factorization of analytic functions with values in non-commutative $L_{1}$ spaces and applications, Canad. J. Math. 41 (1989), 882-906.

[15] Q. Xu, Inégalités pour les martingales de Hardy et renormage des espaces quasi-normés, C.R. Acad. Paris 306(1988), 601-604.

[16] Q. Xu, Convexité uniforme et inégalités de martingales Math. Ann. 287(1990), 193-211.

[17] Q. Xu, Littlewood-Paley theory for functions with values in uniformly convex spaces, J. reine angew. Math. 504(1998), 195-226.

Departamento de Matemáticas Universidad de Valencia

46100 Burjassot

Valencia

Spain

oblasco@uv.es
Matematicki fakultet

11001 Belgrade, pp 550

Serbia

Yugoslavia

pavlovic@poincare.matf.bg.ac.yu 